International Journal of Pure and Applied Mathematics

Volume 84 No. 1 2013, 1-12

ISSN: 1311-8080 (printed version); ISSN: 1314-3395 (on-line version)

url: http://www.ijpam.eu

doi: http://dx.doi.org/10.12732/ijpam.v84i1.1

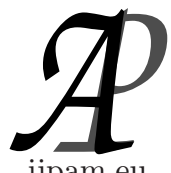

ijpam.eu

\title{
OSCILLATION RESULTS FOR NONLINEAR SECOND ORDER MATRIX DIFFERENTIAL SYSTEMS WITH DAMPING
}

\author{
Wenying Shi ${ }^{1}$, Shaoqin $\mathrm{Gao}^{2}$, Wensheng Zhao ${ }^{3}$ \\ ${ }^{1,2}$ College of Mathematics and Computer Science \\ Hebei University \\ Baoding, Hebei, 071002, P.R. CHINA \\ ${ }^{3}$ Computer Center \\ Hebei Normal University of Science and Technology \\ Qinhuangdao, Hebei, 066004, P.R. CHINA
}

Abstract: In this paper, we investigate the oscillation of second order nonlinear matrix differential system with damping

$$
\left[R(t) Y^{\prime}(t)\right]^{\prime}+P(t) Y^{\prime}(t)+F\left(t, Y(t), Y^{\prime}(t)\right)=0, \quad t \geq t_{0} .
$$

By using a matrix Riccati typetransformation and matrix inequalities, some new oscillation criteria for the system are obtained.

Our results are not contained in known results.

Key Words: oscillation, matrix differential systems, dampingm non-linearity

\section{Introduction}

Consider the second order nonlinear matrix differential system with damped term

$$
\left[R(t) Y^{\prime}(t)\right]^{\prime}+P(t) Y^{\prime}(t)+F\left(t, Y(t), Y^{\prime}(t)\right)=0, \quad t \geq t_{0},
$$

where $R(t), P(t), Y(t)$ and $F\left(t, Y(t), Y^{\prime}(t)\right)$ are $n \times n$ matrices of real valued continuous functions on the interval $\left[t_{0}, \infty\right)$, and $R(t)$ is symmetric and positive definite, $P(t)$ is symmetric. By $M^{T}$ we mean the transpose of the matrix $M$.

Received: July 5, 2012

(c) 2013 Academic Publications, Ltd.

$\S$ Correspondence author url: www.acadpubl.eu 
Hereafter we assume that:

$\left(\mathbf{H}_{1}\right)$ there exists a $n \times n$ real symmetric matrix $Q(t)$, such that

$$
F\left(t, U(t), U^{\prime}(t)\right) U^{-1}(t) \geq Q(t), \quad t \geq t_{0}
$$

in which $U(t)$ is a $n \times n$ nonsingular continuous derivative matrix function in $\left[t_{0}, \infty\right)$.

A solution $Y(t)$ of $(1.1)$ is said to be nontrivial solution if $\operatorname{det} Y(t) \neq 0$ for at least one $t \in\left[t_{0}, \infty\right)$. A nontrivial solution $Y(t)$ of (1.1) is said to be prepared if $Y(t)$ satisfies

$$
\begin{aligned}
& \left(Y^{\prime}(t)\right)^{T} R(t) Y(t)=(Y(t))^{T} R(t) Y^{\prime}(t), \\
& \left(Y^{\prime}(t)\right)^{T} P(t) Y(t)=(Y(t))^{T} P(t) Y^{\prime}(t),
\end{aligned}
$$

i.e., $(Y(t))^{T} R(t) Y^{\prime}(t)$ and $(Y(t))^{T} P(t) Y^{\prime}(t)$ are symmetric. A prepared solution $Y(t)$ of (1.1) is called to be oscillatory if det $Y(t)$ has arbitrary large zeroes. system (1.1) is called to be oscillatory if its every prepared solution is oscillatory.

The oscillatory solution properties for (1.1) with its special case:

$$
Y^{\prime \prime}(t)+Q(t) Y(t)=0
$$

and

$$
\left[R(t) Y^{\prime}(t)\right]^{\prime}+Q(t) Y(t)=0
$$

are important in the mechanical systems associated with (1.1). Therefore, such properties have been studied quite extensively, and some criteria for oscillation of systems (1.4) and (1.5) have been established (see [1-12]).

Recently, Sun and Meng [13,14] using a positive linear functional have established some oscillation criteria for the linear matrix differential syatem with damped

$$
\left[P(t) Y^{\prime}(t)\right]^{\prime}+R(t) Y^{\prime}(t)+Q(t) Y(t)=0 .
$$

However, all existing oscillation results for linear matrix differential systems (1.4)-(1.6) generally cannot be applied to nonlinear matrix differential system (1.1).

In this paper, we will further investigate the system (1.1) and present some new oscillation criteria for system (1.1).

For convenience, we introduce the following notation. Let $\Re$ be the linear space of $n \times n$ real matrices, $\Re_{0} \subset \Re$ be the subspace of symmetric matrices. For any real symmetric matrices $A, B, C \in \Re_{0}$, we write $A \geq B$ to mean that $A-B \geq 0$, that is, $A-B$ is positive semi-definite and $A>B$ to mean that 
$A-B>0$, that is, $A-B$ is positive definite. We will use some properties of this ordering, viz.,

(1) If $A \geq B$, then $\int_{a}^{b} A d t \geq \int_{a}^{b} B d t$;

(2)If $A>0$, then $A^{-1}>0$, and there exists nonsingular symmetric matrix $M$ such that $M^{2}=A$.

Definition. Let $L$ be a linear functional on $\Re, L$ is called "positive" if:

(i) $L(A)>0$ for any $A \in \Re_{0}$ and $A>0$,

(ii) $L(B) \geq 0$ for any $B \in \Re_{0}$ and $B \geq 0$.

Lemma 1.1. (see [14]) If $L$ be a positive linear functional on $\Re$, then for all $A \in \Re, B \in \Re_{0}$ with $B>0$,

$$
L\left[B^{-1}\right] L\left[A^{T} B A\right] \geq(L[A])^{2} .
$$

\section{Main Results}

In the sequel, we say that a function $u=u(t)$ belongs to a function class $D(a, b)=\left\{u \in C^{1}[a, b]: u(t) \not \equiv 0, u(a)=u(b)=0\right\}$, Denoted by $u \in D(a, b)$.

Lemma 2.1. Assume that $\left(H_{1}\right)$ holds. If there exist functions $u \in D(a, b)$, $\rho \in C^{1}\left(\left[t_{0}, \infty\right),(0, \infty)\right)$ and $f \in C^{1}\left(\left[t_{0}, \infty\right), R\right)$, such that

$$
\begin{aligned}
\int_{a}^{b} u^{2}(t) G(t) d t & \\
& >\int_{a}^{b} a(t) u^{\prime}(t)\left\{u^{\prime}(t) R(t)-u(t)\left[P(t)-\rho^{-1}(t) \rho^{\prime}(t) R(t)\right]\right\} d t,
\end{aligned}
$$

where $[a, b] \subset\left[t_{0}, \infty\right), a(t)=\rho(t) \exp \left\{-2 \int_{t_{0}}^{t} f(s) d s\right\}$,

$$
\begin{aligned}
G(t)= & a(t)\left\{Q-f(t) P(t)+f^{2}(t) R(t)-[f(t) R(t)]^{\prime}\right\} \\
& -\frac{1}{4} a(t)\left[P(t) R^{-1}(t) P(t)-2 \rho^{-1}(t) \rho^{\prime}(t) P(t)+\left(\rho^{-1}(t) \rho^{\prime}(t)\right)^{2} R(t)\right] .
\end{aligned}
$$

Then for every prepared solution $Y(t)$ of system (1.1), det $Y(t)$ has at least one zero in $[a, b]$.

Proof. Suppose the contrary, that $Y(t)$ is a prepared solution of system (1.1) and $\operatorname{det} Y(t) \neq 0$ for all $t \in[a, b]$. Let

$$
V(t)=a(t)\left\{R(t) Y^{\prime}(t) Y^{-1}(t)+f(t) R(t)\right\}, \quad t \in[a, b],
$$


then from (1.2) and $R(t)=R^{T}(t)$, we have that $V(t)$ is symmetric. From (1.1), we have

$$
\begin{aligned}
V^{\prime}(t)= & a^{\prime}(t)\left\{R(t) Y^{\prime}(t) Y^{-1}(t)+f(t) R(t)\right\}+a(t)\left\{\left[R(t) Y^{\prime}(t)\right]^{\prime} Y^{-1}(t)\right. \\
& \left.+R(t) Y^{\prime}(t)\left[Y^{-1}(t)\right]^{\prime}+[f(t) R(t)]^{\prime}\right\} \\
= & a^{\prime}(t) a^{-1}(t) V(t)+a(t)\left\{-P(t) Y^{\prime}(t) Y^{-1}(t)-F\left(t, Y(t), Y^{\prime}(t)\right) Y^{-1}(t)\right. \\
& \left.-R(t) Y^{\prime}(t) Y^{-1}(t) Y^{\prime}(t) Y^{-1}(t)+[f(t) R(t)]^{\prime}\right\} \\
= & {\left[\rho^{-1}(t) \rho^{\prime}(t)-2 f(t)\right] V(t)-P(t) R^{-1}(t)[V(t)-a(t) f(t) R(t)] } \\
& -a(t) F\left(t, Y(t), Y^{\prime}(t)\right) Y^{-1}(t) \\
& -a^{-1}(t)[V(t)-a(t) f(t) R(t)] R^{-1}(t)[V(t)-a(t) f(t) R(t)] \\
& +a(t)[f(t) R(t)]^{\prime} \\
= & {\left[\rho^{-1}(t) \rho^{\prime}(t) I_{n}-P(t) R^{-1}(t)\right] V(t)-a^{-1}(t) V(t) R^{-1}(t) V(t) } \\
& +G_{1}(t)-a(t) F\left(t, Y(t), Y^{\prime}(t)\right) Y^{-1}(t),
\end{aligned}
$$

where $G_{1}(t)=a(t)\left\{f(t) P(t)-f^{2}(t) R(t)+[f(t) R(t)]^{\prime}\right\}, I_{n}$ is an $n \times n$ identity matrix.

From (1.3) and $R(t)=R^{T}(t)$, we can get

$$
P(t) Y^{\prime}(t) Y^{-1}(t)=\left(Y^{-1}\right)^{T}\left(Y^{\prime}(t)\right)^{T} P(t),
$$

and then

$$
\begin{aligned}
P(t) R^{-1}(t) V(t) & =a(t)\left\{P(t) Y^{\prime}(t) Y^{-1}(t)+f(t) P(t)\right\} \\
& =a(t)\left\{\left[Y^{-1}(t)\right]^{T}\left[Y^{\prime}(t)\right]^{T} P(t)+f(t) P(t)\right\} \\
& =V^{T}(t) R^{-1}(t) P(t) \\
& =V(t) R^{-1}(t) P(t),
\end{aligned}
$$

i.e., $P(t) R^{-1}(t) V(t)$ is symmetric.

Set

$$
U(t)=V(t)+\frac{1}{2} a(t)\left[P(t)-\rho^{-1}(t) \rho^{\prime}(t) R(t)\right],
$$

then $U(t)$ is symmetric, and noticing that (2.4), we have

$$
\begin{aligned}
a^{-1}(t) & U(t) R^{-1}(t) U(t) \\
= & a^{-1}(t)\left\{V(t)+\frac{a(t)}{2}\left[P(t)-\rho^{-1}(t) \rho^{\prime}(t) R(t)\right]\right\} \\
& R^{-1}(t)\left\{V(t)+\frac{a(t)}{2}\left[P(t)-\rho^{-1}(t) \rho^{\prime}(t) R(t)\right]\right\}
\end{aligned}
$$




$$
\begin{aligned}
= & a^{-1}(t) V(t) R^{-1}(t) V(t)+\frac{1}{2}\left[V(t) R^{-1}(t) P(t)+P(t) R^{-1}(t) V(t)\right] \\
& -\rho^{-1}(t) \rho^{\prime}(t) V(t) \\
& +\frac{1}{4} a(t)\left[P(t)-\rho^{-1}(t) \rho^{\prime}(t) R(t)\right] R^{-1}(t)\left[P(t)-\rho^{-1}(t) \rho^{\prime}(t) R(t)\right] \\
= & a^{-1}(t) V(t) R^{-1}(t) V(t)+\left[P(t) R^{-1}(t)-\rho^{-1}(t) \rho^{\prime}(t) I_{n}\right] V(t) \\
& +\frac{1}{4} a(t)\left[P(t) R^{-1}(t) P(t)-2 \rho^{-1}(t) \rho^{\prime}(t) P(t)+\left(\rho^{-1}(t) \rho^{\prime}(t)\right)^{2} R(t)\right] .
\end{aligned}
$$

From (2.3) and (2.6), we obtain

$$
V^{\prime}(t)=-a^{-1}(t) U(t) R^{-1}(t) U(t)+G_{2}(t)-a(t) F\left(t, Y(t), Y^{\prime}(t)\right) Y^{-1}(t),
$$

where

$$
G_{2}(t)=G_{1}(t)+\frac{1}{4} a(t)\left[P(t) R^{-1}(t) P(t)-2 \rho^{-1}(t) \rho^{\prime}(t) P(t)+\left(\rho^{-1}(t) \rho^{\prime}(t)\right)^{2} R(t)\right] .
$$

From $\left(H_{1}\right)$ and $(2.7)$, we obtain

$$
G(t) \leq-V^{\prime}(t)-a^{-1}(t) U(t) R^{-1}(t) U(t)
$$

where

$$
\begin{aligned}
G(t)= & a Q-G_{2} \\
= & a(t)\left\{Q-f(t) P(t)+f^{2}(t) R(t)-[f(t) R(t)]^{\prime}\right\} \\
& -\frac{1}{4} a(t)\left[P(t) R^{-1}(t) P(t)-2 \rho^{-1}(t) \rho^{\prime}(t) P(t)+\left(\rho^{-1}(t) \rho^{\prime}(t)\right)^{2} R(t)\right] .
\end{aligned}
$$

Multiplying both sides of (2.8) by $u^{2}(t)$ and integrating on $[a, b]$, using the condition $u(a)=u(b)=0$ and (2.5), we obtain

$$
\begin{aligned}
\int_{a}^{b} & u^{2}(t) G(t) d t \\
\leq & -\int_{a}^{b} u^{2}(t) V^{\prime}(t) d t-\int_{a}^{b} u^{2}(t) a^{-1}(t) U(t) R^{-1}(t) U(t) d t \\
= & \int_{a}^{b} 2 u(t) u^{\prime}(t) V(t) d t-\int_{a}^{b} u^{2}(t) a^{-1}(t) U(t) R^{-1}(t) U(t) d t \\
= & \int_{a}^{b} 2 u(t) u^{\prime}(t) U(t) d t-\int_{a}^{b} u^{2}(t) a^{-1}(t) U(t) R^{-1}(t) U(t) d t \\
& -\int_{a}^{b} u(t) u^{\prime}(t) a(t)\left[P(t)-\rho^{-1}(t) \rho^{\prime}(t) R(t)\right] d t
\end{aligned}
$$


Since $R(t)>0$ (i.e., $R(t)$ is positive definite), then $R^{-1}(t)>0$ and there exists a nonsingular symmetric matrix $M(t)$, such that $M^{2}(t)=R^{-1}(t)$, it follows that from (2.9), we have

$$
\begin{aligned}
& \int_{a}^{b} u^{2}(t) G(t) d t \\
& \leq \int_{a}^{b} 2 u(t) u^{\prime}(t) M^{-1}(t)[M(t) U(t) M(t)] M^{-1}(t) d t \\
&-\int_{a}^{b} u^{2}(t) a^{-1}(t) M^{-1}(t)[M(t) U(t) M(t)]^{2} M^{-1}(t) d t \\
&-\int_{a}^{b} u(t) u^{\prime}(t) a(t)\left[P(t)-\rho^{-1}(t) \rho^{\prime}(t) R(t)\right] d t \\
&=-\int_{a}^{b} M^{-1}(t)\left\{\sqrt{a^{-1}(t)} u(t)[M(t) U(t) M(t)]-\sqrt{a(t)} u^{\prime}(t) I_{n}\right\}^{2} M^{-1}(t) d t \\
&+\int_{a}^{b} a(t) u^{\prime 2}(t) R(t) d t-\int_{a}^{b} u(t) u^{\prime}(t) a(t)\left[P(t)-\rho^{-1}(t) \rho^{\prime}(t) R(t)\right] d t \\
& \leq+\int_{a}^{b} a(t) u^{\prime 2}(t) R(t) d t-\int_{a}^{b} u(t) u^{\prime}(t) a(t)\left[P(t)-\rho^{-1}(t) \rho^{\prime}(t) R(t)\right] d t \\
&= \int_{a}^{b} a(t) u^{\prime}(t)\left\{u^{\prime}(t) R(t)-u(t)\left[P(t)-\rho^{-1}(t) \rho^{\prime}(t) R(t)\right]\right\} d t,
\end{aligned}
$$

which contradicts the assumption (2.1). The proof is complete.

From Lemma 2.1, it is easy to see that the following Theorem 2.2 is true.

Theorem 2.2. Assume that $\left(H_{1}\right)$ holds. If for each $T \geq t_{0}$, there exist $a, b \in R$ with $T \leq a<b$, and $u \in D(a, b), \rho \in C^{1}\left(\left[t_{0}, \infty\right),(0, \infty)\right)$ and $f \in$ $C^{1}\left(\left[t_{0}, \infty\right), R\right)$, such that (2.1) holds, then system (1.1) is oscillatory.

Theorem 2.3. Assume that $\left(H_{1}\right)$ holds. If there exist functions $\rho \in$ $C^{1}\left(\left[t_{0}, \infty\right),(0, \infty)\right), f \in C^{1}\left(\left[t_{0}, \infty\right), R\right)$ and a positive linear functional $L$ on $\Re$ such that for any $t_{1} \geq t_{0}$,

$$
\lim _{t \rightarrow \infty} \int_{t_{1}}^{t} \frac{d s}{a(s) L[R(s)]}=\infty,
$$

and

$$
\lim _{t \rightarrow \infty} L\left[J\left(t, t_{1}\right)\right]=\infty,
$$


where

$$
\begin{aligned}
a(t)= & \rho(t) \exp \left\{-2 \int_{t_{0}}^{t} f(s) d s\right\}, \\
J\left(t, t_{1}\right) & =\frac{1}{2} a(t)\left[\rho^{-1}(t) \rho^{\prime}(t) R(t)-P(t)\right]+\int_{t_{1}}^{t} B(s) d s \\
B(t)= & a(t)\left\{Q-f(t) P(t)+f^{2}(t) R(t)-[f(t) R(t)]^{\prime}\right\} \\
& -\frac{1}{4} a(t)\left[P(t) R^{-1}(t) P(t)-2 \rho^{-1}(t) \rho^{\prime}(t) P(t)+\left(\rho^{-1}(t) \rho^{\prime}(t)\right)^{2} R(t)\right] .
\end{aligned}
$$

Then system (1.1) is oscillatory.

Proof. Suppose to the contrary that there exists a prepared solution $Y(t)$ of (1.1) such that $\operatorname{det} Y(t) \neq 0$ on $\left[t_{1}, \infty\right)$ for some $t_{1} \geq t_{0}$. Let

$$
V(t)=-a(t)\left\{R(t) Y^{\prime}(t) Y^{-1}(t)+f(t) R(t)\right\}, \quad t \geq t_{1},
$$

and

$$
U(t)=V(t)-\frac{1}{2} a(t)\left[P(t)-\rho^{-1}(t) \rho^{\prime}(t) R(t)\right], \quad t \geq t_{1} .
$$

Similar to the proof of (2.7) in Lemma 2.1, we obtain

$$
V^{\prime}(t)=a^{-1}(t) U(t) R^{-1}(t) U(t)+B_{2}(t)+a(t) F\left(t, Y(t), Y^{\prime}(t)\right) Y^{-1}(t),
$$

where

$$
\begin{gathered}
B_{2}(t)=B_{1}(t)-\frac{1}{4} a(t)\left[P(t) R^{-1}(t) P(t)-2 \rho^{-1}(t) \rho^{\prime}(t) P(t)+\left(\rho^{-1}(t) \rho^{\prime}(t)\right)^{2} R(t)\right], \\
B_{1}(t)=a(t)\left\{f^{2}(t) R(t)-f(t) P(t)-[f(t) R(t)]^{\prime}\right\} .
\end{gathered}
$$

From (2.15) and noting that $\left(H_{1}\right)$, we obtain

$$
V^{\prime}(t) \geq a^{-1}(t) U(t) R^{-1}(t) U(t)+B(t)
$$

where

$$
\begin{aligned}
B(t)= & B_{2}(t)+a(t) Q(t) \\
= & a(t)\left\{Q+f^{2}(t) R(t)-f(t) P(t)-[f(t) R(t)]^{\prime}\right\} \\
& -\frac{1}{4} a(t)\left[P(t) R^{-1}(t) P(t)-2 \rho^{-1}(t) \rho^{\prime}(t) P(t)+\left(\rho^{-1}(t) \rho^{\prime}(t)\right)^{2} R(t)\right] .
\end{aligned}
$$

Integrating both sides of (2.16) from $t_{1}$ to $t$, we obtain

$$
V(t) \geq V\left(t_{1}\right)+\int_{t_{1}}^{t} B(s) d s+\int_{t_{1}}^{t} a^{-1}(s) U(s) R^{-1}(s) U(s) d s .
$$


¿From (2.17) and (2.14), we obtain

$$
U(t) \geq V\left(t_{1}\right)+J\left(t, t_{1}\right)+\int_{t_{1}}^{t} a^{-1}(s) U(s) R^{-1}(s) U(s) d s,
$$

where $J\left(t, t_{1}\right)=\frac{1}{2} a(t)\left[\rho^{-1}(t) \rho^{\prime}(t) R(t)-P(t)\right]+\int_{t_{1}}^{t} B(s) d s$.

Since $R(t)>0$, we obtain, by applying $L$ to (2.18),

$$
\begin{aligned}
L[U(t)] & \geq L\left[V\left(t_{1}\right)\right]+L\left[J\left(t, t_{1}\right)\right]+L\left[\int_{t_{1}}^{t} a^{-1}(s) U(s) R^{-1}(s) U(s) d s\right] \\
& \geq L\left[V\left(t_{1}\right)\right]+L\left[J\left(t, t_{1}\right)\right] .
\end{aligned}
$$

By (2.12), there exists a $t_{2}>t_{1}$ such that $L\left[V\left(t_{1}\right)\right]+L\left[J\left(t, t_{1}\right)\right]>0$ for $t \geq t_{2}$. Hence

$$
L[U(t)]>0, \quad t \geq t_{2}
$$

and

$$
L[U(t)]>L\left[\int_{t_{1}}^{t} a^{-1}(s) U(s) R^{-1}(s) U(s) d s\right], \quad t \geq t_{2} .
$$

Define

$$
W(t)=\int_{t_{1}}^{t} a^{-1}(s) U(s) R^{-1}(s) U(s) d s, \quad t \geq t_{2},
$$

then for $t \geq t_{2}$, we have

(i) $L[U(t)]>L[W(t)]$,

(ii) $L[W(t)]=\int_{t_{1}}^{t} a^{-1}(s) L\left[U(s) R^{-1}(s) U(s)\right] d s$ $\geq \int_{t_{1}}^{t} \frac{L([U(s)])^{2}}{a(s) L[R(s)]} d s>0 \quad$ (by Lemma 1.1 and (2.20)),

(iii) $(L[W(t)])^{\prime}=L\left[W^{\prime}(t)\right]=L\left[a^{-1}(t) U(t) R^{-1}(t) U(t)\right]$

$$
\begin{gathered}
\geq \frac{(L[U(t)])^{2}}{a(t) L[R(t)]} \quad(\text { by Lemma 1.1) } \\
>\frac{(L[W(t)])^{2}}{a(t) L[R(t)]} \quad(\text { by }(2.22)) .
\end{gathered}
$$

Hence

$$
\frac{1}{a(t) L[R(t)]}<\frac{(L[W(t)])^{\prime}}{(L[W(t)])^{2}}, \quad t \geq t_{2} .
$$


Integrating both sides of the above inequality from $t_{2}$ to $t$, we obtain

$$
\int_{t_{2}}^{t} \frac{1}{a(s) L[R(s)]} d s<\frac{1}{L\left[W\left(t_{2}\right)\right]}-\frac{1}{L[W(t)]}<\frac{1}{L\left[W\left(t_{2}\right)\right]},
$$

which is contradiction to (2.11). Hence system (1.1) is oscillatory.

If we choosing $f(t)=0, \rho(t)=1$ in Theorem 2.2 and Theorem 2.3, then we have the follow Corollaries.

Corollary 2.4. Assume that $\left(H_{1}\right)$ holds. If for each $T \geq t_{0}$, there exist $a, b \in R$ with $T \leq a<b$, and $u \in D(a, b)$ such that

$$
\begin{aligned}
\int_{a}^{b} u^{2}(t)\left[Q(t)-\frac{1}{4} P(t) R^{-1}(t) P(t)\right] d t & \\
& >\int_{a}^{b} u^{\prime}(t)\left[u^{\prime}(t) R(t)-u(t) P(t)\right] d t,
\end{aligned}
$$

then system (1.1) is oscillatory.

Corollary 2.5. Assume that $\left(H_{1}\right)$ holds. If there exist a positive linear functional $L$ on $\Re$ such that for any $t_{1} \geq t_{0}$,

$$
\lim _{t \rightarrow \infty} \int_{t_{1}}^{t} \frac{d s}{L[R(s)]}=\infty
$$

and

$$
\lim _{t \rightarrow \infty} L\left[\int_{t_{1}}^{t}\left\{Q(s)-\frac{1}{4} P(s) R^{-1}(s) P(s)\right\} d s-\frac{1}{2} P(t)\right]=\infty
$$

then system (1.1) is oscillatory.

In order to illustrate our results, we consider the following example.

Example. Consider the second order matrix differential system

$$
\left[R(t) Y^{\prime}(t)\right]^{\prime}+P(t) Y^{\prime}(t)+F\left(t, Y(t), Y^{\prime}(t)\right)=0, \quad t \geq 1,
$$

where $R(t)=t^{2} I_{n}, P(t)=2 t I_{n}, F\left(t, Y, Y^{\prime}\right)=\operatorname{diag}\left(\alpha_{1} t+1, \alpha_{2} t+1, \cdots, \alpha_{n} t+\right.$ 1) $Y+Y Y^{T} Y\left(\alpha_{i}, i=1,2, \ldots, n\right.$ are constants). It is easy to verify that

$$
\begin{aligned}
F\left(t, Y, Y^{\prime}\right) Y^{-1} & =\operatorname{diag}\left(\alpha_{1} t+1, \alpha_{2} t+1, \cdots, \alpha_{n} t+1\right)+Y Y^{T} \\
& \geq \operatorname{diag}\left(\alpha_{1} t+1, \alpha_{2} t+1, \cdots, \alpha_{n} t+1\right) \equiv Q(t) .
\end{aligned}
$$


Let $u(t)=\sin \sqrt{t}$. For any $T \geq 1$, choose $k$ sufficiently large so that $(2 k \pi)^{2} \geq T$ and set $a=(2 k \pi)^{2}, b=(2 k+1)^{2} \pi^{2}$ in (2.23). It is easy to verify that

$$
\begin{aligned}
& u^{2}(t)\left[Q(t)-\frac{1}{4} P(t) R^{-1}(t) P(t)\right]-u^{\prime}(t)\left[u^{\prime}(t) R(t)-u(t) P(t)\right] \\
&= \sin ^{2} \sqrt{t}\left[Q(t)-I_{n}\right]-\frac{t}{4} \cos ^{2} \sqrt{t} I_{n}+\sqrt{t} \sin \sqrt{t} \cos \sqrt{t} I_{n} \\
&= \frac{1}{2}(1-\cos 2 \sqrt{t}) \operatorname{diag}\left(\alpha_{1} t, \alpha_{2} t, \cdots, \alpha_{n} t\right)-\frac{t}{8}(1+\cos 2 \sqrt{t}) I_{n} \\
&+\frac{\sqrt{t}}{2} \sin 2 \sqrt{t} I_{n} \\
&= \frac{t}{2} \operatorname{diag}\left(\alpha_{1}, \alpha_{2}, \cdots, \alpha_{n}\right)-\frac{t}{2} \cos 2 \sqrt{t} \operatorname{diag}\left(\alpha_{1}, \alpha_{2}, \cdots, \alpha_{n}\right)-\frac{t}{8} I_{n} \\
&-\frac{t}{8} \cos 2 \sqrt{t} I_{n}+\frac{\sqrt{t}}{2} \sin 2 \sqrt{t} I_{n} \\
&= \frac{t}{8} \operatorname{diag}\left(4 \alpha_{1}-1,4 \alpha_{2}-1, \cdots, 4 \alpha_{n}-1\right) \\
&-\frac{t}{8} \cos 2 \sqrt{t} \operatorname{diag}\left(4 \alpha_{1}+1,4 \alpha_{2}+1, \cdots, 4 \alpha_{n}+1\right)+\frac{\sqrt{t}}{2} \sin 2 \sqrt{t} I_{n} .
\end{aligned}
$$

Taking integral from a to $b$, using integration by parts, and noting that

$$
\int_{(2 k \pi)^{2}}^{(2 k+1)^{2} \pi^{2}} t \cos 2 \sqrt{t} d t=0, \quad \int_{(2 k \pi)^{2}}^{(2 k+1)^{2} \pi^{2}} \sqrt{t} \sin 2 \sqrt{t} d t=0
$$

we have

$$
\begin{aligned}
\int_{a}^{b}\left\{u^{2}(t)\right. & {\left.\left[Q(t)-\frac{1}{4} P(t) R^{-1}(t) P(t)\right]-u^{\prime}(t)\left[u^{\prime}(t) R(t)-u(t) P(t)\right]\right\} d t } \\
& =\int_{(2 k \pi)^{2}}^{(2 k+1)^{2} \pi^{2}} \frac{t}{8} \operatorname{diag}\left(4 \alpha_{1}-1,4 \alpha_{2}-1, \cdots, 4 \alpha_{n}-1\right) d t \\
& =\operatorname{diag}\left(4 \alpha_{1}-1,4 \alpha_{2}-1, \cdots, 4 \alpha_{n}-1\right) \int_{(2 k \pi)^{2}}^{(2 k+1)^{2} \pi^{2}} \frac{t}{8} d t
\end{aligned}
$$

Since $\int_{(2 k \pi)^{2}}^{(2 k+1)^{2} \pi^{2}} \frac{t}{8} d t>0$, hence, when $\alpha_{i}>1 / 4, i=1,2, \cdots, n$, we have

$$
\operatorname{diag}\left(4 \alpha_{1}-1,4 \alpha_{2}-1, \cdots, 4 \alpha_{n}-1\right) \int_{(2 k \pi)^{2}}^{(2 k+1)^{2} \pi^{2}} \frac{t}{8} d t>0
$$

i.e., (2.23) holds. Applying Corollary 2.4, we find that (2.26) is oscillatory for $\alpha_{i}>1 / 4, i=1,2, \cdots, n$.

Remark 1. It is easy to see that the results in [1-14] are not applicable to equation (2.26) since $P(t) \neq 0$ or $F\left(t, Y, Y^{\prime}\right) \neq Q(t) Y$. 


\section{Acknowledgments}

Supported by the NNSF of P.R. China (10971045, 11271106), the NSF of Hebei Province of China (A2009000151).

\section{References}

[1] D.B. Hinton, R.T. Lewis, Oscillation theory of generalized second order differential equations, Rocky Mountain J.Math., 10, No. 2 (1980), 751-761.

[2] R. Byers, B.J. Harris, M.K. Kwong, Weighted means and Oscillation conditions for second order matrix differential equations, J. Diff. Eqs., 61, No. 1 (1986), 164-177.

[3] G.J. Butler, L.H. Erbe, Oscillation results for self-adjoint differential systems, Mathl. Anal. Appl., 115 (1986), 470-481.

[4] G.J. Butler, L.H. Erbe, A.B. Mingarelli, Riccati techniques and variational principles in Oscillation theory for linear systems, Tran. Amer. Math. Soc., 303, No. 1 (1987), 263-282.

[5] N. Parhi, P. Praharaj, Oscillation of linear second order matrix differential equations, Mathl. Anal. Appl., 211 (1998), 287-305.

[6] L.H. Erbe, Q. Kong, S. Ruan, Kamenev type theorems for second order matrix differential systems, Proc. Amer. Math. Soc, 117, No. 1 (1993), 957-962.

[7] M.K. Kwong, H.G. Kaper, K. Akiyama, A.B. Mingarelli, Oscillation of linear second-order differential systems, Proc. Am. Math. Soc., 91 (1984), 85-91.

[8] Z.W. Zheng, F.W. Meng, Y.H. Yu, Oscillation of second order matrix differential systems, Acta. Math. Sinica., 41, No. 6 (1998), 1231-1238 (In Chinese).

[9] R.K. Zhuang, Interval criteria for oscillatory of second order matrix differential systems, Acta. Math. Sinica., 44, No. 6 (2001), 1037-1044 (In Chinese).

[10] Q.R. Wang, X.M. Wu, S.M. Zhu, Kamenev-Type Oscillation criteria for second-order matrix differential systems, Appl. Mathl. lett., 16 (2003), 821826. 
[11] Q.R. Wang, Oscillation of self-adjoint matrix differential systems, Appl. Mathl. lett., 17 (2004), 1299-1305.

[12] F.W. Meng, J. Wang, Z. Zheng, A note on Kamenev type theorems for second order matrix differential systems, Proc. Am. Math. Soc., 126 (1998), 391-395.

[13] Y.G. Sun, F.W. Meng, Oscillation results for matrix differential systems with damping, Appl. Mathl. Comput., 170 (2005), 545-555.

[14] Y.G. Sun, F.W. Meng, Oscillation results for matrix differential systems with damping, Appl. Mathl. Comput., 175 (2006), 212-220.

[15] X.J. Yang, Oscillatory criteria for certain second-order matrix differential equations, Mathl. Anal. Appl., 265 (2002), 285-295.

[16] Q. Yang, Y. Tang, Oscillation theorems for certain second order self-adjoint matrix differential systems, Mathl. Anal. Appl., 288 (2003), 565-585. 